\title{
Multiparametric autoimmune diagnostics: recent advances
}

This article was published in the following Dove Press journal:

Pathology and Laboratory Medicine International

27 April 2016

Number of times this article has been viewed

\section{Jan Damoiseaux}

Central Diagnostic Laboratory, Maastricht University Medical Centre, Maastricht, the Netherlands
Correspondence: Jan Damoiseaux

Central Diagnostic Laboratory, Maastricht University Medical Centre, P Debyelaan 25, 6229HX Maastricht, the Netherlands

Tel +3I 433876655

Email jan.damoiseaux@mumc.nl
Abstract: Autoimmune diagnostics in a routine clinical laboratory is constantly challenged by the discovery of new autoantibodies and technical innovations in the immunoassays applied. These challenges are, in particular, combined in the multiparametric immunoassays. Appropriate positioning of multiparametric immunoassays within the laboratory requires integrated knowledge of the clinical performance of the test system for each individual antigen, the conditions prescribed in disease criteria and/or guidelines, and the demands of the clinicians. This review provides a summary of the multiparametric immunoassays available, as well as the applications and restrictions in routine clinical practice.

Keywords: autoantibodies, line immunoassay, dot immunoassay, addressable laser bead immunoassay, indirect immunofluorescence

\section{Introduction}

The presence of autoantibodies is a hallmark of many autoimmune diseases. The detection of these autoantibodies, being either pathogenic or just a biomarker for the presence of disease, has appeared very helpful in the diagnostic workup of many autoimmune diseases. The number of autoantigens identified as targets for autoantibodies is steadily increasing over the years. ${ }^{1}$ This involves not only autoantibodies in diseases that nowadays are newly recognized as autoimmune diseases, but also autoantibodies that enable the identification of subgroups of well-known autoimmune diseases. For a long time, antigen specificity of autoantibodies has been primarily determined in monospecific assays, such as enzyme-linked immunoassays (ELISA) and radioimmunoassays (RIA). In cases where identification of multiple antibodies is relevant for a group of related diseases, screening by indirect immunofluorescence (IIF) on cells or tissues is considered an efficient first step..$^{2-4}$ This approach is even considered the gold standard for systemic autoimmune rheumatic diseases (SARD) and antineutrophil cytoplasmic antibody (ANCA)-associated vasculitis (AAV). ${ }^{5,6}$ For SARD, the presence of antinuclear antibodies (ANA) is determined by IIF on HEp-2 cells. If positive, most laboratories analyze the reactivities with multiple distinct extractable nuclear antigens (ENA), either directly in monospecific assays or first by prescreening with a mixture of the respective ENA. ${ }^{7}$ Clearly, combining these monospecific assays in a multiparametric assay has many logistic advantages. Due to the expanding repertoire of autoantibodies, this approach has become increasingly relevant for many other autoimmune diseases, but clearly may also have its downsides. 
Multiparametric assays are defined as immunoassays that enable the unequivocal identification of autoantibody reactivity toward a multitude of single antigens. Such assays include addressable laser bead immunoassay (ALBIA) and line or dot immunoassay (LIA/DIA). If an assay is based on a mixture of known and/or unknown antigens, for instance IIF or Western blotting, this is not considered a multiparametric immunoassay because the identity of the recognized antigen is not unequivocally revealed. On the other hand, novel IIF assays that enable the detection of multiple monospecific antigens are within the definition of multiparametric assays.

In this review, first, the methodologies of the distinct multiparametric assays will be described. This description is restricted to assays that are readily available for routine diagnostic laboratories; experimental array technologies are excluded. Second, the applicability of multiparametric technology for diagnosis of autoimmune diseases will be discussed. Finally, the restrictions of the multiparametric approach will be reviewed in order to increase the awareness that test characteristics of each individual antigen should be known for appropriate interpretation in the clinical context of the patient under investigation.

\section{Distinct methods of multiparametric immunoassays}

The ALBIA employs a mixture of antigen-labeled beads with a unique internal fluorescent signature emitted by the beads (Figure 1A). Therefore, each bead population represents a separate immunoassay. Upon incubation with the serum sample, the respective autoantibodies will bind to the beads, and the nonspecific antibodies are removed by a washing step. Next, the beads are incubated with fluorescent antihuman immunoglobulin reagent, and the presence of autoantibodies is evaluated by flow cytometry. Using autoantibody standards of different concentrations, a calibration curve can be composed from the signal intensities, and the curve is then used to determine the autoantibody concentrations in the test samples. Because international reference material is still lacking for most autoantibodies, results are generally reported as arbitrary units. Altogether, ALBIA enables to differentially quantitate the presence of multiple autoantibodies at the same time. This method can be fully automated either on a single platform or a dual platform and therefore is suited for high throughput.

The second type of multiparametric immunoassays includes LIA and DIA. These assays are often, erroneously, referred to as line or dot blots. However, in both cases, antigen is transferred directly to the adsorbent membrane without prior electrophoresis and blotting (Figure 1B). In the case of DIA, the antigen is spotted on the membrane, while in the case of LIA, the antigen is "painted" as a straight line on the membrane. In contrast to Western blotting, both DIA and LIA enable the use of distinct binding conditions for each separate antigen to the membrane. Also the choice for native or recombinant antigens is free for each antigen. Separate pieces of membrane, containing different antigens, can be mounted on a carrier synthetic foil. The strip is incubated with serum of the patient. Depending on the presence of antibodies for the respective antigens on the strip, antibody-antigen complexes will form. These complexes can be visualized with antihuman immunoglobulin reagent conjugated to an enzyme and subsequent conversion of the appropriate substrate into detectable products at the sites of protein immobilization. The incubation procedure can be automated, although several systems require manual pipetting of the patient sample. Therefore, this technique is not optimal for high volume testing, but instead is very convenient for laboratories that have low-to-medium sample numbers. Reading of results can be automated, and the color intensities of the bands/dots can be evaluated to enable the semiquantitative detection of autoantibodies.

Although IIF was originally based on the detection of autoantibodies binding to cells or tissues, new developments have enabled the combination of the original approach with antigen-specific tests (Figure 1C). The CytoBead method (Generic Assays, Berlin, Germany) combines the screening of autoantibodies to cellular substrates with a multiparametric microparticle immunoassay. The beads coated with different antigens can be identified by the size as well as location in one out of four compartments surrounding the central part of the well containing the cellular substrate. ${ }^{8,9}$ The Biochip method (Euroimmun, Lübeck, Germany) also enables the combination of multiple substrates in a single incubation field. These substrates may consist of any combination of cells, tissues, antigen dots, or transfected cells. ${ }^{10}$ In particular, the transfected cells have appeared very suitable for the detection of autoantibodies to membrane receptors that heavily depend on their proper integration in the lipid bilayer for correct presentation of the immunogenic epitopes. For the detection of autoantibodies by IIF, serum samples are incubated with antigen substrate on microscope slides to allow specific binding of autoantibodies. The antigen substrate may either be air-dried or prepared with fixative to enable autoantibody binding. After washing to remove nonspecific antibodies, the glass slide is incubated with an antihuman immunoglobulin reagent conjugated to fluorescein 

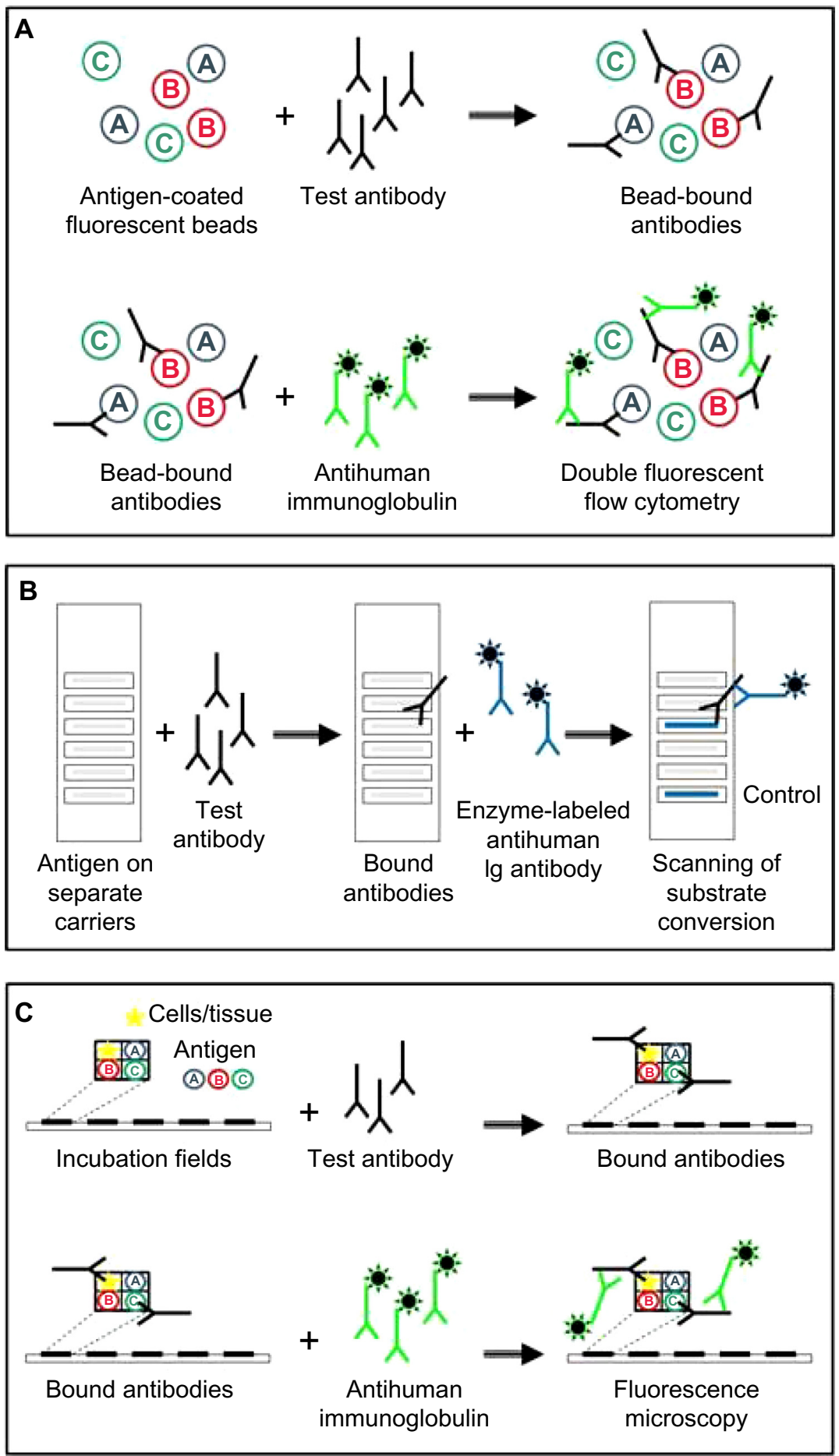

Figure I Distinct methods of multiparametric immunoassays.

Notes: The methods that are currently available in routine diagnostic laboratories are visualized: addressable laser bead immunoassay (A), line and dot immunoassay (B), and indirect immunofluorescence $(\mathbf{C})$. All assays are based on first-step incubation with patient serum, second-step incubation with antihuman immunoglobulin conjugate, and, in case of line and dot immunoassay (B), third-step detection of the reaction product.

isothiocyanate. The complete incubation procedure can be automated in robots specifically designed for microscope slides. The final three-part complex, consisting of fluorescent secondary antibody, autoantibody, and antigen, can be visualized with the aid of a fluorescent microscope. The readout of
IIF is subjective and requires an experienced microscopist. Results obtained by IIF can be quantitated (semiquantitative) by testing serial, two-step dilutions or performing quantitative image analysis of the serum samples. The latter technique quantitates fluorescence of a patient sample in comparison to 
the intensity of standardized calibrators and directly converts intensity into an antibody titer. More recently, pattern recognition devices have become available that enable automated reading of these multiparametric IIF assays. ${ }^{8,9,11}$ If fully automated, medium-to-high volume testing can be handled. In case of the CytoBead method, the ANCA specificity assays even generate quantitative results by utilizing a standard curve based on reference sera of the International Center for Disease Control and Prevention (CDC). ${ }^{9}$

Basically, multiparametric assays for autoantibody detection can be applied for any autoimmune disease or cluster of related autoimmune diseases that is associated with more than a single autoantibody. These diseases, and their respective autoantigens, are summarized in Tables 1 and 2 . For some diseases, multiparametric assays are (not yet) available. The first reason for this might be that the distinct antigens do not allow optimal detection in a single assay format. This holds in particular for diabetes mellitus (DM) type I, myasthenia gravis (MG), and thyroid autoimmune diseases where at least one of the clinically relevant antigens, ie, glutamic acid decarboxylase (GAD)65, acetyl-choline receptor (AChR), and thyroid stimulating hormone receptor (TSH-R), respectively, requires a methodology not compatible with currently available multiparametric assays. In this respect, it is to be mentioned that several multiparametric assays utilize recombinant antigens that do not fully represent the epitope repertoire of native antigens. This is most apparent for the three autoantigens mentioned, but may also hold for many other autoantigens applied in autoantibody detection assays. Clearly, this problem is not restricted to multiparametric assays. Second, rheumatoid arthritis (RA) relies on autoantibody detection in monospecific tests because the autoantibodies included in the classification criteria are of different isotypes, ie, IgM rheumatoid factor and IgG anti-citrullinated protein antibodies. ${ }^{12}$ Although it is technically possible to apply detection reagents differentially conjugated with distinct fluorochromes in flow cytometry (ALBIA) and fluorescence microscopy (IIF), such applications are not readily available in routine settings. The diagnostic need for detecting autoantibodies of distinct isotypes is not restricted to RA, but also holds for the antiphospholipid syndrome (APS), the Guillain-Barré and Miller-Fisher syndrome (GBS/MFS), and, to a lesser extent, celiac disease. In particular, APS and GBS/MFS would benefit from the simultaneous detection of distinct autoantibody isotypes. Finally, the currently available IIF assays that enable the detection of multiple autoantibodies in celiac disease and thyroid autoimmune diseases do not fulfill the definition of multiparametric assays because in both cases one autoantibody is detected in tissue and another

Table I Overview of systemic autoimmune diseases that require detection of multiple autoantibodies

\begin{tabular}{|c|c|c|c|}
\hline Disease & Autoantigens & Technologies & Comment \\
\hline AAV & MPO, PR3, GBM & $\begin{array}{l}\text { ALBIA, LIA/DIA, } \\
\text { IIF }\end{array}$ & $\begin{array}{l}\text { Anti-GBM is optional because of overlap in clinical } \\
\text { presentation; the consensus requires a two-step approach, } \\
\text { which is combined in the IIF multiplex assays }\end{array}$ \\
\hline APS & Cardiolipin, $\beta 2-G P I$ & ALBIA, LIA/DIA & $\begin{array}{l}\text { LIA/DIA may contain additional phospholipids and protein } \\
\text { cofactors; the APS consensus requires separate detection of } \\
\text { IgM and IgG autoantibodies, }{ }^{35} \text { while the SLICC criteria for SLE } \\
\text { also require detection of IgA autoantibodies }{ }^{26}\end{array}$ \\
\hline IIM & $\begin{array}{l}\text { Jo-I, PL-7, PL-12, EJ, OJ, Ha, Zo, KS, } \\
\text { PM-Scl, Ku, Mi-2, MDA-5, TIFI- } \gamma \text {, } \\
\text { NXP2, SAE, SRP, HMGCR }\end{array}$ & LIA/DIA & $\begin{array}{l}\text { IIM is to be considered a disease that requires a distinct } \\
\text { testing approach than the other SARD. The identification } \\
\text { of the antigen specificity enables to differentiate between } \\
\text { distinct subtypes of IIM }\end{array}$ \\
\hline RA & lgG, citrullinated proteins & NA & $\begin{array}{l}\text { The clinically relevant isotype is different for both } \\
\text { autoantibodies (IgM RF and IgG ACPA) }\end{array}$ \\
\hline SARD & $\begin{array}{l}\text { Sm, RNP, SSA, SSB, TRIM2I, CENP-B, } \\
\text { Topo I, Jo-I, PCNA, PM-Scl, RibP, }\end{array}$ & $\begin{array}{l}\text { ALBIA, LIA/DIA, } \\
\text { IIF }\end{array}$ & $\begin{array}{l}\text { Especially LIA/DIA may contain additional IIM and SSc-related } \\
\text { autoantibodies }\end{array}$ \\
\hline
\end{tabular}
DFS70, dsDNA, nucleosomes, histones

SSc CENP-A/B, Topo I, PM-Scl, RNA LIA/DIA polymerase III, Ku, fibrillarin, NOR90, Th/To, PDGFR

Only CENP-B, Topo I, and RNA polymerase III are part of the classification criteria; ${ }^{31}$ interpretation of other specificities is hampered by low prevalence and limited state of validation

Abbreviations: AAV, ANCA-associated vasculitis; ACPA, anti-citrullinated protein antibodies; ALBIA, addressable laser bead immunoassay; APS, anti-phospholipid syndrome; DIA, dot immunoassay; IIF, indirect immunofluorescence; IIM, idiopathic inflammatory myopathies; LIA, line immunoassay; NA, not available; RA, rheumatoid arthritis; RF, rheumatoid factor; SARD, systemic autoimmune rheumatic diseases; SSc, systemic sclerosis; SLICC, Systemic Lupus International Collaborating Clinics; SLE, systemic lupus erythematosus; Ig, immunoglobulin. 
Table 2 Overview of organ-specific autoimmune diseases that require detection of multiple autoantibodies

\begin{tabular}{|c|c|c|c|}
\hline Disease & Autoantigens & Technologies & Comment \\
\hline AIG/PA & $\mathrm{H}+/ \mathrm{K}+\mathrm{ATPase}, \mathrm{IF}$ & LIA/DIA & IIF detects APCA reactivity in gastric tissue and IF on antigen dots \\
\hline $\mathrm{AlH} / \mathrm{PBC}$ & $\begin{array}{l}\text { F-actin, cyt P450 IID6 (LKMI), } \\
\text { M2/PDC, SLA, LCI, gP2I0, } \\
\text { spI00 }\end{array}$ & LIA/DIA, IIF & $\begin{array}{l}\text { Autoantibodies to the distinct subunits of PDC may be identified; } \\
\text { also autoantibodies to additional antigens may be detected }\end{array}$ \\
\hline Celiac disease & ENDA, tTG, DG & ALBIA, IIF & $\begin{array}{l}\text { ALBIA detects tTG and DG, while IIF detects endomysial reactivity } \\
\text { in esophagus tissue and DG on antigen dots; autoantibodies in } \\
\text { celiac disease are of IgA isotype, but increased prevalence of } \lg A \\
\text { deficiency requires additional testing for lgG isotype }\end{array}$ \\
\hline DM type I & GAD65, IA2, IAA, ZNT8 & NA & \\
\hline GBS/MFS & GMI-4, GDI-3, GT/QI & LIA/DIA & Separate detection of $\lg M$ and $\lg G$ autoantibodies is required \\
\hline $\begin{array}{l}\text { Limbic } \\
\text { encephalitis }\end{array}$ & $\begin{array}{l}\text { NMDA-R, AMPAI/2-R, GABA-R, } \\
\text { VGKC (CASPR2, LGII), DPPX, } \\
\text { IgLON5 }\end{array}$ & IIF & $\begin{array}{l}\text { Detection of these autoantibodies requires localization of the } \\
\text { antigen in a lipid bilayer; hence, transfected cells are used as } \\
\text { substrate; the list of relevant antigens is still expanding }\end{array}$ \\
\hline MG & AChR, MuSK, LRP4, titin & NA & Anti-titin antibodies may identify patients with MG with a thymoma \\
\hline $\begin{array}{l}\text { Pemphigus/ } \\
\text { pemphigoid }\end{array}$ & Dsgl/3, BPI80/230 & IIF & $\begin{array}{l}\text { Anti-plakin autoantibodies are in particular relevant for } \\
\text { paraneoplastic pemphigus }\end{array}$ \\
\hline PNS & $\begin{array}{l}\mathrm{Hu}, \text { Yo, } \mathrm{Ri}, \mathrm{CV} 2, \mathrm{Mal} / 2 \text {, amphiphysin, } \\
\text { recoverin, SOXI, titin, Zic4, GAD65, } \\
\operatorname{Tr} \text { (DNER) }\end{array}$ & LIA/DIA & $\begin{array}{l}\text { The list of relevant antigens is still expanding (for instance: PKC } \gamma \text {, } \\
\text { CARP VII, Ca/ARHGAP26) }\end{array}$ \\
\hline
\end{tabular}

Thyroid AID

$\mathrm{Tg}$, TPO, TSH-R

NA

IIF enables detection of thyroid microsome reactivity, suggestive for TPO, in thyroid tissue and $\mathrm{Tg}$ on antigen dots

Abbreviations: AID, autoimmune disease; AIG, autoimmune gastritis; AlH, autoimmune hepatitis; ALBIA, addressable laser bead immunoassay; APCA, anti-parietal cell antibodies; DIA, dot immunoassay; DM, diabetes mellitus; GBS, Guillain-Barré syndrome; IIF, indirect immunofluorescence; LIA, line immunoassay; MFS, Miller-Fisher syndrome; MG, myasthenia gravis; NA, not available; PA, pernicious anemia; PBC, primary biliary cirrhosis; PDC, pyruvate dehydrogenase complex; PNS, paraneoplastic neurological syndrome.

on antigen-specific dots. Therefore, these applications will not be further elaborated upon.

\section{Multiparametric autoantibody diagnostics in systemic autoimmune diseases}

Application of multiparametric technology is most apparent in the systemic autoimmune diseases (Table 1). For AAV, the international consensus for ANCA detection prescribes screening with IIF on ethanol-fixed neutrophils and, if positive, antigen-specific testing for myeloperoxidase (MPO) and proteinase 3 (PR3). ${ }^{6}$ Although, because of improvement of the antigen-specific assays, the necessity of IIF screening is being disputed, ${ }^{13}$ multiparametric IIF very elegantly combines the cellular- and antigen-specific substrates and thereby follows the international consensus. ${ }^{911,14}$ The improvements achieved in the detection of MPO- and PR3-ANCA are primarily based on the methodology used for coating the antigen to the solid phase. Binding of the antigen via a capturing monoclonal antibody, ie, second-generation immunoassay, or via a peptide-linker, ie, third-generation immunoassay, has been proven superior to direct coating of the antigen, ie, first-generation immunoassay. ${ }^{3}$ Interestingly, none of the currently available multiparametric assays utilizes second- or third-generation methodologies. Since AAV may present with clinical manifestations overlapping with the Goodpasture's syndrome, ie, rapidly progressive glomerulonephritis and/ or alveolar hemorrhage, the simultaneous detection of antibodies to the glomerular basement membrane (GBM) can be indicated. Most multiparametric assays, therefore, enable detection of autoantibodies to all the three antigens: MPO, PR3, and GBM. Since clinical indications for ANCA testing are much wider than those for anti-GBM testing, it is questionable if this is cost-effective, also when taking into account that autoantibody detection is used for follow-up of disease. ${ }^{15}$ Simultaneous detection, though, can be detrimental if disease presentation has a rapidly progressive onset with life-threatening organ damage, and short turn-around-time results are required. For diagnostic purposes quantitative values for autoantibodies are less important, but follow-up of disease benefits from quantitative results which are not readily available from all multiparametric methods.

SARD are a group of rheumatic diseases that may have overlapping clinical manifestations, in particular at the onset of the disease. Hence, identification of antigenspecific autoantibodies may aid in the differential diagnosis. Traditionally, SARD include systemic lupus erythematosus (SLE), Sjögren's syndrome (SjS), systemic sclerosis (SSc), idiopathic inflammatory myopathies (IIM), and mixed connective tissue disease (MCTD). IIF, on HEp-2 cells, is still 
the method of choice as a screening assay for ANA. ${ }^{5}$ As is the case in AAV, also in SARD there is ongoing discussion whether IIF is the optimal choice for all types of SARD. ${ }^{16}$ In particular, in a primary care setting, the low pretest probability of SARD in combination with the low specificity of ANA IIF may hamper appropriate clinical interpretation of a positive result. ${ }^{17}$ Furthermore, for IIM, the sensitivity of ANA detection by IIF is limited, and due to the discovery of multiple other autoantigens (Tables 1 and 2), first-step application of IIM-specific multiparametric assays seems more appropriate. ${ }^{18-21}$ As such, diagnostic testing for IIM should not be incorporated in the testing algorithm for SARD. ${ }^{1}$ However, this requires early clinical recognition of IIM as a separate entity and, subsequently, close collaboration between the clinician and the laboratory specialist. ANA detection by IIF for $\mathrm{SjS}$ is part of the most recent classification criteria, ${ }^{22}$ but is not optimal for the detection of anti-SSA antibodies. ${ }^{23}$ The multiparametric CytoBead assay circumvents this shortcoming by combining the cellular- and antigen-specific substrates. ${ }^{8}$ In general, multiparametric assays are used as a second, or even third step in the testing algorithm for SARD. Following a positive ANA IIF result, multiparametric assays enable direct identification of possible antigen-specific antibodies. Since not all potential ANA antigens have been identified, a screening assay with a mixture of multiple ENA may be applied in between. In particular, ALBIA enables the simultaneous application of both ENA screening and ENA identification. In some laboratories, ENA identification is confirmed in an independent (monospecific) assay. In these algorithms, the composition of the SARDrelated autoantigens, as being present in the multiparametric assay, is of great importance. As already discussed earlier, IIM-associated autoantigens are of limited value since the presence of only Jo-1 suggests that autoantibody detection for IIM is sufficiently covered. Some multiparametric assays for SARD include multiple IIM-associated autoantigens, but because of the low incidence of IIM this may not be cost-effective and reduces the specificity of the assay. For the other SARD, multiparametric assays are of added value as long as the autoantigen repertoire is adequately covered. The classification criteria for SLE include antibodies to both dsDNA as well as $\mathrm{Sm}(\mathrm{D}) .{ }^{24-26}$ Solid-phase assays for anti-dsDNA antibodies, however, are not considered the first choice. ${ }^{5}$ Although in SLE a wide array of autoantibodies have been described, ${ }^{27}$ most of these autoantibodies are not clinically relevant in daily clinical practice. Additional autoantibodies that are clinically important are directed to nucleosomes and ribosomal $\mathrm{P}$ proteins (RibP). Detection of anti-PCNA antibodies is often considered to be strongly associated with SLE, ${ }^{28}$ but this association more recently appeared to be quite limited. ${ }^{29}$ One antigen that is consistently lacking in the SARD-related multiparametric assays is C1q. Anti-C1q antibodies in SLE are indicative of lupus nephritis. ${ }^{30}$ However, testing for anti-C1q antibodies requires special conditions to prevent aspecific binding of circulating immune complexes and/or immunoglobulin aggregates. Detection of anti-SSA antibodies is of help for the diagnosis of chronic discoid lupus erythematosus. In case of MCTD, high levels of anti-RNP antibodies are a hallmark of disease. This clearly requires semiquantitative detection of anti-RNP antibodies. Furthermore, since anti-RNP antibodies may be directed to distinct components of the RNP/Sm complex, a decision tree should be available for correct interpretation if these components are separately present in the multiparametric assay. The classification criteria for systemic sclerosis have recently been updated. ${ }^{31}$ Besides autoantibodies to CENP-B and topoisomerase I (Scl-70), autoantibodies to RNA polymerase III have also been included. Although the arguments for this addition can be disputed, ${ }^{32}$ the criteria require inclusion of these autoantibodies in the testing algorithm. Since most multiparametric assays for SARD do not yet include RNA polymerase III, different strategies for monospecific detection of autoantibodies to RNA polymerase III can be envisaged. ${ }^{33}$ Alternatively, similar to the suggested approach for IIM, a multiparametric assay for the whole repertoire of SSc-associated antibodies (Tables 1 and 2) can be applied. ${ }^{34}$ Altogether, differential testing algorithms for SARD are depicted in Figure 2.

The classification criteria for APS encompass two autoantibodies, ie, anti-cardiolipin antibody (aCLA) and anti- $\beta 2$-glycoprotein I ( $\beta 2$-GPI) antibody, ${ }^{35}$ that can be detected by conventional immunoassays. The third autoantibody, lupus anticoagulant (LAC), can be detected in a multistep coagulation assay. ${ }^{36}$ The aCLA and anti- $\beta 2-$ GPI antibodies, as being part of the APS criteria, are of $\operatorname{IgM}$ and IgG isotype. ${ }^{35}$ Since there is a strong overlap between APS and SLE, these antibodies are also part of the classification criteria for SLE. ${ }^{24-26}$ However, in the SLICC criteria also IgA is mentioned as a relevant isotype. ${ }^{26}$ This implies that for both antigen specificities 2-3 test incubations are further indicated. Prescreening with a total immunoglobulin conjugate and, if positive, second step testing for specific isotypes may be an alternative approach. As mentioned, multiparametric assays that enable differential detection of distinct isotypes would be most optimal but are not available yet. In the ongoing search for other autoantigens in APS, multiple 




Figure 2 Testing algorithm for systemic autoimmune rheumatic diseases.

Notes: The method of choice for first-step testing is ANA IIF. In case of a positive result, a second-step identification of autoantibody reactivity is required. Primary care screening benefits from performing an anti-ENA screen as first step; if suspicion of SjS is indicated, combining ANA IIF with anti-ENA screen is recommended as first step, and if suspicion of IIM is indicated, multiparametric testing for IIM-associated autoantibodies is recommended as first step. Furthermore, in case of suspicion of SSc, a positive ANA IIF result is preferentially followed by multiparametric testing for SSc-associated autoantibodies. Assays within solid rectangles indicate first-step assays, assays within bold rectangles indicate that multiparametric testing is available, while assays within dashed rectangles are optional.

Abbreviations: ANA, antinuclear antibodies; ENA, extractable nuclear antigens; IIF, indirect immunofluorescence; IIM, idiopathic inflammatory myopathies; MCTD, mixed connective tissue disease; SARD, systemic autoimmune rheumatic diseases; SjS, Sjögren's syndrome; SLE, systemic lupus erythematosus; SSc, systemic sclerosis.

negatively charged phospholipids, differentially combined with coagulation-associated cofactors, have been identified. These autoantibodies can be tested in LIA/DIA, ${ }^{37}$ but these tests will only reveal qualitative results. Since the APS classification criteria request that the autoantibody levels are above the 99th percentile of a healthy control population, the cutoff control of LIA/DIA should be set as such. Correct clinical interpretation of results obtained in these extended multiparametric assays for APS remains a challenge.

\section{Multiparametric autoantibody diagnostics in organ-specific autoimmune diseases}

Besides the systemic autoimmune diseases, organ-specific autoimmune diseases also seem to be increasingly fit for multiparametric autoantibody diagnostics (Table 2). IIF on tissue sections and/or cells is, most often, still the screening method of choice because it enables to evaluate the presence or absence of multiple distinct autoantibodies directed to native proteins or multimeric complexes. In case of a positive result, reflex testing with multiparametric testing may be applied to confirm the antigen specificity of the autoantibodies. Also, in case of organ-specific autoimmune diseases, the combination of IIF on tissues/cells and antigen-specific analysis nowadays is readily available.

Several autoimmune diseases affecting the gastrointestinal tract may benefit from multiparametric autoantibody detection. These diseases include autoimmune gastritis (AIG), pernicious anemia (PA), autoimmune hepatitis $(\mathrm{AIH})$, primary biliary cirrhosis $(\mathrm{PBC})$, and celiac disease. Traditionally, the respective autoantibodies are detected by IIF on tissues, ie, stomach, liver, kidney, and esophagus. At least for celiac disease, identification of the relevant autoantigens has reversed the diagnostic approach of autoantibody detection. Screening for autoantibodies should start by detecting $\operatorname{IgA}$ autoantibodies to tissue transglutaminase (tTG), and positive results are to be confirmed by endomysial staining of esophagus tissue by IIF. ${ }^{38}$ Multiparametric technology for celiac disease is only applicable if additional autoantibodies, like anti-deamidated gliadin antibodies, are included. Since IgA deficiency is highly prevalent in celiac disease, many laboratories combine IgA tTG with IgG 
anti-deamidated gliadin antibody testing. ${ }^{39}$ As mentioned before, multiparametric assays do not yet allow the use of distinct isotype-specific reagents simultaneously. Also, the relevant autoantigens related to $\mathrm{AIG}, \mathrm{AIH}$, and $\mathrm{PBC}$ have been identified. Nevertheless, in many laboratories, IIF on tissue blocks (stomach, liver, and kidney) is still the preferred screening option for these autoantibodies. The advantage is that distinct autoantibodies can be identified in the same assay. On the other hand, the presence of anti-mitochondrial antibodies (AMA) may, for instance, obscure the presence of anti-parietal cell antibodies (APCA). Furthermore, if a patient is suspected, for instance, of PBC and the IIF test coincidentally reveals the presence of APCA, one has to decide whether to ignore or report this result. Nowadays, several distinct multiparametric modalities have become available for these diseases. These LIA/DIA enable unequivocal detection of autoantibodies to the relevant antigens, while the distinct disease entities, ie, AIG/PA and AIH/PBC, can be approached with separate assays. A restriction of this approach is the limited availability of F-actin, the main autoantigen that is recognized in type $1 \mathrm{AIH}$, and revealing smooth muscle cell staining by IIF. Furthermore, the diagnostic criteria for AIH differentially score the presence of autoantibodies based on the titer obtained by IIF. ${ }^{40}$ Also, a consensus statement from the committee for autoimmune serology of the International Autoimmune Hepatitis Group states that the basic technique for the routine testing of autoantibodies relevant to AIH is IIF on multiorgan substrates, including kidney, liver, and stomach. ${ }^{41}$ Multiparametric IIF assays, combining multiorgan substrates with antigen-specific substrates, however, are in development.

With respect to the neuronal autoimmune diseases, the last decade has revealed a multitude of new autoantigen specificities. ${ }^{1}$ This holds both for the paraneoplastic neurologic syndrome (PNS) as well as the autoimmune encephalopathies (Table 2). Multiparametric autoantibody assays, therefore, are detrimental for the diagnostic workup of these diseases. In case of PNS, screening for the presence of relevant antibodies can be performed by IIF on cerebellum tissue. Since recognition of all relevant staining patterns is difficult, multiparametric LIA/DIA are most often applied in parallel. A similar approach is advised for the autoimmune encephalopathies: IIF on hippocampus in combination with multiparametric identification of the autoantibody reactivity. The autoantigens are merely membrane receptors that only maintain the relevant conformational epitopes when integrated in a lipid bilayer. The method of choice is a cell-based IIF assay. Differential transfection of eukaryotic cells with the respective DNA enables detection of multiple autoantibody reactivity in the biochip method. ${ }^{42}$ Finally, multiparametric assays are also the most convenient for detection of autoantibodies to distinct types of gangliosides for the diagnosis of the GBS/MFS. ${ }^{43}$ These anti-ganglioside autoantibodies, though, have to be analyzed both for the IgM and IgG isotype separately.

The last organ-specific autoimmune disease that benefits from multiparametric immunoassays entails the spectrum of blistering skin diseases. ${ }^{4,44}$ Detection of tissue-bound or circulating autoantibodies against structural components maintaining cell-cell and cell-matrix interactions is essential for diagnosis of these diseases. ${ }^{45}$ Again, the combination of screening on tissue, ie, esophagus and/or salt-split skin, with identification on antigen-specific substrates enables adequate detection of the relevant autoantibodies..$^{10}$ The antigens can be exposed in transfected cells, ie, the cell-based assay, or as antigen dots, all combined in the biochip IIF method. The variant dermatitis herpetiformis, however, is considered a subtype of celiac disease and therefore the diagnostic algorithm for autoantibody detection in celiac disease is most appropriate.

\section{Limitations of multiparametric autoantibody diagnostics}

The first major issue of consideration with respect to multiparametric immunoassays is the composition of the autoantigen repertoire in the assay. To prevent unwanted coincidental findings, the autoantigen repertoire should be limited to a single disease or a combination of clinically related diseases. As far as diagnostic and/or classification criteria exist, at least the autoantibodies included in these criteria should be detectable in the assay. This implies that a multiparametric assay for SSc is not restricted to CENP-B and topoisomerase I, but also includes RNA polymerase III. ${ }^{31}$ In this respect, it should be noted that technical developments by definition precede inclusion in criteria. For instance, incorporation of anti-citrullinated protein antibodies in the criteria for RA took $>10$ years, ${ }^{12,46}$ while the criteria for AIH still include detection of anti-smooth muscle cell antibodies by IIF, but not autoantibodies to F-actin. ${ }^{40,41}$ Next, it has to be decided whether the assay should only enable detection of the most prevalent autoantibodies or the whole repertoire of autoantigens identified. If the choice is made for the most prevalent autoantibodies, a multiparametric assay is of limited added value if all the relevant antigens are not available. In particular, in many LIA/DIA techniques for AIH/ $\mathrm{PBC}, \mathrm{F}$-actin is lacking and therefore still requires testing 
with alternative techniques. Furthermore, in the first option SSc patients the clinician has to be aware that the suspected disease may not be excluded by a negative result, while the second option increases the risk of false-positive results. Clearly, the specificity of a multiparametric immunoassay decreases by the number of autoantigens that is available in the assay. Being complete, however, brings us to another downside of multiparametric immunoassays. Given the still growing spectrum of autoantibodies in some diseases, like the neuronal autoimmune diseases, it is a real challenge for the diagnostic companies to keep up with all new developments and to maintain good quality of the test system. Especially, if it concerns extremely rare autoantibody specificities, reliable quality assurance may be at stake. An example is the detection of autoantibodies to the PDGF receptor in SSc. According to the first description of these autoantibodies, nearly all patients with SSc should test positive in a functional assay.$^{47}$ Incorporation of this antigen in the Euroimmun SSc LIA revealed positive results in only $1 \%$ of the patients with SSc. The questions to be asked here are if the relevant antigenic epitopes are adequately expressed in the SSc LIA and if these autoantibodies can only be detected in functional assays? Also for the laboratory specialist, multiparametric immunoassays that contain the autoantigens recognized by low-prevalent autoantibodies are challenging. Ever increasing requirements for laboratory accreditation, such as ISO15189, ask for extensive validation of the diagnostic assays and participation in external quality control. For adequate validation, sufficient serum samples with the respective autoantibodies are often lacking and external quality control programs, most often for logistic reasons, focus on the most prevalent autoantibodies. As such, it is interesting that flexible multiparametric assays start to enter the market. The Blue Diver Combi test (D-tek, Mons, Belgium) is a flexible test that enables a free choice of up to six antigens in a stripholder for DIA. Although less flexible, the composition of biochips (Euroimmun) can be custom-based. In the end, the laboratory specialist is responsible for correct integration and interpretation of the multiparametric assays within the autoimmune laboratory. Diagnostic performance may improve if the reactivity toward two related components, for instance, PM/Scl-75 and PM/Scl-100, or RNA polymerase III RP11 or RP155 in $\mathrm{SSc}$, is combined, or if insufficiently validated reactivity, for instance, to fibrillarin or $\mathrm{Th} / \mathrm{To}$, is not reported. ${ }^{48}$

\section{Conclusion}

Multiparametric immunoassays are widely introduced in the diagnostic workup of SARD and, in particular, of IIM and SSc, since these two diseases are associated with a wide array of autoantibodies. IIF, however, also enables to identify antigen-specific autoantibodies, even in combination with cellular and/or tissue substrates. Importantly, automation of these IIF assays is in progress. In IIF, the autoantigen may be present on beads, as dots, or in the membranes of transfected cells. The latter cell-based assay has appeared detrimental for the diagnosis of autoimmune encephalopathies. One major concern of introducing multiparametric immunoassays in the laboratory is the difficulty to validate the test for rare autoantibodies and the lack of external quality control programs for these autoantibodies. It is evident that for appropriate validation, in particular of the rare entities, large multicenter studies are required. Furthermore, a number of autoimmune diseases benefit from quantification of autoantibody levels for diagnosis and/or follow-up and this is not readily available for all multiparametric immunoassays. Finally, the development of multiparametric immunoassays that are able to simultaneously measure autoantibodies of distinct isotypes will further enhance the applicability of these assays in the autoimmune laboratory.

\section{Disclosure}

The author reports no conflicts of interest in this work.

\section{References}

1. Damoiseaux J, Andrade LE, Fritzler MJ, Shoenfeld Y. Autoantibodies 2015: from diagnostic biomarkers toward prediction, prognosis and prevention. Autoimmun Rev. 2015;14:555-563.

2. Damoiseaux JG, Tervaert JW. From ANA to ENA: how to proceed? Autoimmun Rev. 2006;5:10-17.

3. Cohen Tervaert JW, Damoiseaux J. Antineutrophil cytoplasmic autoantibodies: how are they detected and what is their use for diagnosis, classification and follow-up? Clin Rev Allergy Immunol. 2012;43: 211-219.

4. Damoiseaux J. Bullous skin diseases: classical types of autoimmune diseases. Scientifica (Cairo). 2013;2013:457982.

5. Agmon-Levin N, Damoiseaux J, Kallenberg C, et al. International recommendations for the assessment of autoantibodies to cellular antigens referred to as anti-nuclear antibodies. Ann Rheum Dis. 2014;73:17-23.

6. Savige J, Gillis D, Benson E, et al. International consensus statement on testing and reporting of antineutrophil cytoplasmic antibodies (ANCA). Am J Clin Pathol. 1999;111:507-513.

7. Damoiseaux J, Agmon-Levin N, Van Blerk M, et al. From ANAscreening to antigen-specificity: an EASI-survey on the daily practice in European countries. Clin Exp Rheumatol. 2014;32:539-546.

8. Scholz J, Grossmann K, Knütter I, et al. Second generation analysis of antinuclear antibody (ANA) by combination of screening and confirmatory testing. Clin Chem Lab Med. 2015;53:1991-2002.

9. Sowa M, Grossmann K, Knütter I, et al. Simultaneous automated screening and confirmatory testing for vasculitis-specific ANCA. PLoS One. 2014;9:e107743.

10. Damoiseaux J, van Rijsingen M, Warnemünde N, Dähnrich C, Fechner K, Tervaert JW. Autoantibody detection in bullous pemphigoid: clinical evaluation of the EUROPLUS ${ }^{\mathrm{TM}}$ Dermatology Mosaic. $J$ Immunol Methods. 2012;382:76-80. 
11. Krause C, Ens K, Fechner K, et al. EUROPattern Suite technology for computer-aided immunofluorescence microscopy in autoantibody diagnostics. Lupus. 2015;24:516-529.

12. Aletaha D, Neogi T, Silman AJ, et al. 2010 rheumatoid arthritis classification criteria: an American College of Rheumatology/European League Against Rheumatism collaborative initiative. Ann Rheum Dis. 2010;62:2569-2581.

13. Vermeersch P, Vervaeke S, Blockmans D, et al. Determination of antineutrophil cytoplasmic antibodies in small vessel vasculitis: comparative analysis of different strategies. Clin Chim Acta. 2008;397:77-81.

14. Damoiseaux J, Steller U, Buschtez M, et al. EUROPLUS ANCA BIOCHIP mosaic: PR3 and MPO antigen microdots improve the laboratory diagnostics of ANCA-associated vasculitis. J Immunol Methods. 2009;348:67-73.

15. Rutgers A, Heeringa P, Damoiseaux JG, Cohen Tervaert JW. ANCA and anti-GBM antibodies in diagnosis and follow-up of vasculitic disease. Eur J Int Med. 2003;14:287-295.

16. Mahler M, Meroni PL, Bossuyt X, Fritzler MJ. Current concepts and future directions for the assessment of autoantibodies to cellular antigens referred to as anti-nuclear antibodies. J Immunol Res. 2014;2014:315179.

17. Avery TY, van de Cruys M, Austen J, Stals F, Damoiseaux JG. Antinuclear antibodies in daily clinical practice: prevalence in primary, secondary, and tertiary care. J Immunol Res. 2014;2014:401739.

18. Lega JC, Fabien N, Reynaud Q, et al. The clinical phenotype associated with myositis-specific and associated autoantibodies: a meta-analysis revisiting the so-called antisynthetase syndrome. Autoimmun Rev. 2014;13:883-891.

19. Iaccarino L, Ghirardello A, Bettio S, et al. The clinical features, diagnosis and classification of dermatomyositis. J Autoimmun. 2014; 48-49:122-127.

20. Mahler M, Miller FW, Fritzler MJ. Idiopathic inflammatory myopathies and the anti-synthetase syndrome: a comprehensive review. Autoimmun Rev. 2014;13:367-371.

21. Bodoki L, Nagy-Vincze M, Griger Z, Betteridge Z, Szöllösi L, Dankó K. Four dermatomyositis-specific autoantibodies - anti-TIF1 $\gamma$, anti-NXP2, anti-SAE and anti-MDA5 - in adult and juvenile patients with idiopathic inflammatory myopathies in a Hungarian cohort. Autoimmun Rev. 2014;13:1211-1219.

22. Shiboski SC, Shiboski CH, Criswell L, et al. American College of Rheumatology classification criteria for Sjögren's syndrome: a datadriven, expert consensus approach in the Sjögren's International Collaborative Clinical Alliance cohort. Arthritis Care Res (Hoboken). 2012;64:475-487.

23. Bossuyt X, Fieuws S. Detection of antinuclear antibodies: added value of solid phase assay? Ann Rheum Dis. 2014;73:e11.

24. Tan EM, Cohen AS, Fries JF. The 1982 revised criteria for the classification of systemic lupus erythematosus. Arthritis Rheum. 1982;25:1271-1277.

25. Hochberg MC. Updating the American College of Rheumatology revised criteria for the classification of systemic lupus erythematosus. Arthritis Rheum. 1997;40:1725.

26. Petri M, Orbai AM, Alarcón GS, et al. Derivation and validation of the Systemic Lupus International Collaborating Clinics classification criteria for systemic lupus erythematosus. Arthritis Rheum. 2012; 64:2677-2686.

27. ShererY, Gorstein A, Fritzler MJ, Shoenfeld Y. Autoantibody explosion in systemic lupus erythematosus: more than 100 different antibodies found in SLE patients. Semin Arthritis Rheum. 2004;34:501-538.

28. Mahler M, Miyachi K, Peebles C, Fritzler MJ. The clinical significance of autoantibodies to the proliferating cell nuclear antigen (PCNA). Autoimmun Rev. 2012;11:771-775.

29. Vermeersch P, De Beeck KO, Lauwerys BR, et al. Antinuclear antibodies directed against proliferating cell nuclear antigen are not specifically associated with systemic lupus erythematosus. Ann Rheum Dis. 2009;68:1791-1793.
30. Kallenberg CG. Anti-C1q autoantibodies. Autoimmunity Rev. 2008;7: 612-615.

31. van den Hoogen F, Khanna D, Fransen J, et al. 2013 classification criteria for systemic sclerosis: an American College of Rheumatology/European League Against Rheumatism collaborative initiative. Arthritis Rheum. 2013;65:2737-2747.

32. Damoiseaux J. Are autoantibodies to RNA-polymerase III to be incorporated in routine diagnostic laboratory algorithms for systemic autoimmune rheumatic diseases? Ann Rheum Dis. 2014;73:e29.

33. Bonroy C, Smith V, Van Steendam K, et al. The integration of the detection of systemic sclerosis-associated antibodies in a routine laboratory setting: comparison of different strategies. Clin Chem Lab Med. 2013;51:2151-2160.

34. Mehra S, Walker J, Patterson K, Fritzler MJ. Autoantibodies in systemic sclerosis. Autoimmun Rev. 2013;12:340-354.

35. Miyakis S, Lockshin MD, Atsumi T, et al. International consensus statement on an update of the classification criteria for definite antiphospholipid syndrome (APS). J Thromb Haemost. 2006;4:295-306.

36. Pengo V, Tripodi A, Reber G, et al. Update of the guidelines for lupus anticoagulant detection. Subcommittee on Lupus Anticoagulant/ Antiphospholipid Antibody of the Scientific and Standardisation Committee of the International Society on Thrombosis and Haemostasis. J Thromb Haemost. 2009;7:1737-1740.

37. Roggenbuck D, Egerer K, von Landenberg P, et al. Antiphospholipid antibody profiling: time for a new technical approach? Autoimmun Rev. 2012;11:821-826

38. Husby S, Koletzko S, Korponay-Szabó IR, et al. European Society for Pediatric Gastroenterology, Hepatology, and Nutrition guidelines for the diagnosis of coeliac disease. J Pediatr Gastroenterol Nutr. 2012;54:136-160.

39. Oyaert M, Vermeersch P, De Hertogh G, et al. Combining antibody tests and taking into account antibody levels improves serologic diagnosis of celiac disease. Clin Chem Lab Med. 2015;53:1537-1546.

40. Alvarez F, Berg PA, Bianchi FB, et al. International Autoimmune Hepatitis Group Report: review of criteria for diagnosis of autoimmune hepatitis. J Hepatol. 1999;31:929-938.

41. Vergani D, Alvarez F, Bianchi FB, et al. Liver autoimmune serology: a consensus statement from the committee for autoimmune serology of the International Autoimmune Hepatitis Group. J Hepatol. 2004; 41:677-683.

42. Wandinger KP, Klingbeil C, Gneiss C, et al. New serological markers for the differential diagnosis of autoimmune limbic encephalitis. J Lab Med. 2012;35:329-342.

43. Conrad K, Schneider H, Ziemssen T, et al. A new line immunoassay for the multiparametric detection of antiganglioside autoantibodies in patients with autoimmune peripheral neuropathies. Ann NY Acad Sci. 2007;1109:256-264.

44. Otten JV, Hashimoto T, Hertl M, Payne AS, Sitaru C. Molecular diagnosis in autoimmune skin blistering conditions. Curr Mol Med. 2014;14:69-95.

45. Mihai S, Sitaru C. Immunopathology and molecular diagnosis of autoimmune bullous diseases. J Cell Mol Med. 2007;11:462-481.

46. Schellekens GA, de Jong BA, van den Hoogen FH, van de Putte LB, van Venrooij WJ. Citrulline is an essential constituent of antigenic determinants recognized by rheumatoid arthritis-specific autoantibodies. J Clin Invest. 1998;101:273-281.

47. Baroni SS, Santillo M, Bevilacqua F, et al. Stimulatory autoantibodies to the PDGF receptor in systemic sclerosis. $N$ Engl J Med. 2006;354:2667-2676.

48. Bonroy C, Van Praet J, Smith V, et al. Optim ization and diagnostic performance of a single multiparameter lineblot in the serological workup of systemic sclerosis. J Immunol Methods. 2012;379:53-60. 
Pathology and Laboratory Medicine International

Dovepress

\section{Publish your work in this journal}

Pathology and Laboratory Medicine International is a peer-reviewed, open access journal focusing on innovative basic research and translational research related to pathology or human disease. The journal includes original research, updates, case reports, reviews and commentaries on current controversies. The Academic Sponsor

Submit your manuscript here: http://www.dovepress.com/pathology-and-laboratory-medicine-international-journal of this journal is the Chinese American Pathology Association (CAPA). The manuscript management system is completely online and includes a very quick and fair peer-review system. Visit http://www.dovepress.com/testimonials.php to read real quotes from published authors. 